\title{
Adenocarcinoma, Not Otherwise Specified, Arising in Accessory Lacrimal Gland: A Diagnostic Challenge
}

\author{
Tatyana Milman $^{\mathrm{a}}$ Codrin lacob $^{\mathrm{b}}$ Simeon Lauer ${ }^{\mathrm{c}}$ Michael D. Feldman ${ }^{\mathrm{a}}$ \\ Paul J.L. Zhang a
}

a Department of Pathology and Laboratory Medicine, Hospital of the University of Pennsylvania, Perelman School of Medicine, Philadelphia, PA, and Departments of ${ }^{b}$ Pathology, Ophthalmology, and Otolaryngology and 'Ophthalmology, The Mount Sinai Hospital, New York Eye and Ear Infirmary of Mount Sinai, New York, NY, USA

\section{Established Facts}

- Accessory lacrimal glands can occasionally give rise to benign and malignant neoplasms.

- Several patients with adenoid cystic carcinoma and mucoepidermoid carcinoma of the accessory lacrimal gland have been described in the literature.

\section{Novel Insights}

- Adenocarcinoma of accessory lacrimal gland has not been previously reported.

- A diagnostic algorithm, illustrating the approach to classification of this unusual neoplasm, is described.

\section{Keywords}

Accessory lacrimal gland · Gland of Wolfring · Lacrimal gland $\cdot$ Adenocarcinoma - Adenocarcinoma not otherwise specified $\cdot$ Carcinoma $\cdot$ Eyelid

\section{Abstract \\ Neoplasms arising in accessory lacrimal glands are rare. We describe a 33-year-old man with adenocarcinoma arising in the left lower eyelid accessory lacrimal gland. Microscopic evaluation demonstrated an infiltrative neoplasm com-}

posed of mildly to moderately pleomorphic cells with abundant eosinophilic cytoplasm and focal intracytoplasmic vacuoles, arranged predominantly in ductules. Foci of luminal and intracytoplasmic eosinophilic secretory material and occasional mucin were noted. An in situ component was identified in the gland of Wolfring. Though perineural invasion was present, high-grade nuclear features, brisk mitotic activity, and comedonecrosis were not identified. Immunohistochemical studies were notable for immunoreactivity of the tumor cells for CK7, carcinoembryonic antigen, BRST-2, androgen receptors, and HER2/neu (2+). The neo-

\section{KARGER}

(c) 2017 S. Karger AG, Basel

E-Mail karger@karger.com

www.karger.com/oop
Tatyana Milman, MD

Pathology Department, Hospital of the University of Pennsylvania 3400 Spruce Street

Philadelphia, PA 19104 (USA)

E-Mail tatyana.milman@uphs.upenn.edu 
plastic cells were negative for CK20, estrogen and progesterone receptors, $\mathrm{S}-100, \mathrm{p} 63$, calponin, thyroid transcription factor-1, and prostate-specific antigen. Fluorescence in situ hybridization studies for ETV6 and MAML2 rearrangements and for HER2/neu amplification were negative. Because of the absence of unifying morphologic, immunophenotypic, and molecular genetic findings, the diagnosis of adenocarcinoma, not otherwise specified, was rendered. The patient underwent comprehensive oncologic workup, which was negative for another primary tumor and metastases. He remains disease free with a follow-up of 4 years. This case illustrates the challenges encountered in applying salivary gland tumor classification to the accessory lacrimal gland neoplasm.

(c) 2017 S. Karger AG, Basel

\section{Introduction}

Malignant epithelial neoplasms arising in accessory lacrimal glands are extremely rare and present several challenges to both the pathologist and the treating clinician [1-5]. It may be difficult, if not impossible, to localize the tumor unequivocally to the accessory lacrimal gland in the absence of a well-defined in situ component. Additionally, the rarity of accessory lacrimal gland malignancies forces the pathologist to apply the main lacrimal gland or salivary gland classification and staging guidelines to these lesions, which may not be neces- sarily appropriate. Finally, since the biologic behavior of accessory lacrimal gland neoplasms is obscure, the optimal management of these lesions is not well defined. Several patients with well-documented adenoid cystic carcinoma and mucoepidermoid carcinoma of accessory lacrimal gland origin have been, thus far, described in the literature [1-5]. To our knowledge, there have been no prior reports of adenocarcinoma arising in this location. Herein, we describe a patient with adenocarcinoma, not otherwise specified, arising in a gland of Wolfring. The differential diagnosis and the diagnostic studies performed to classify this elusive neoplasm are discussed.

\section{Case Report}

A 33-year-old, otherwise healthy man presented for evaluation of the left lower eyelid lesion. Reportedly, he sustained a chemical burn with sporicide to that area 5 years previously, following which the left lower eyelid was inflamed and irritated, with the mass developing over time. The ophthalmic exam was notable for a firm, erythematous, vascular mass in the central lower eyelid, measuring $20 \mathrm{~mm}$ in greatest dimension, associated with eyelid margin distortion and madarosis (Fig. 1a). The patient underwent a wedge resection of the lower eyelid mass with frozen section control of margins, followed by the reconstruction with Hughes flap.

Gross evaluation of the eyelid wedge resection demonstrated a central lobulated area of tumefaction, most notable at the junction of the tarsal plate and forniceal eyelid tissues (Fig. 1b). Microscopic sections demonstrated an infiltrative neoplasm, which involved diffusely the anterior and posterior lamella of the eyelid and the eyelid
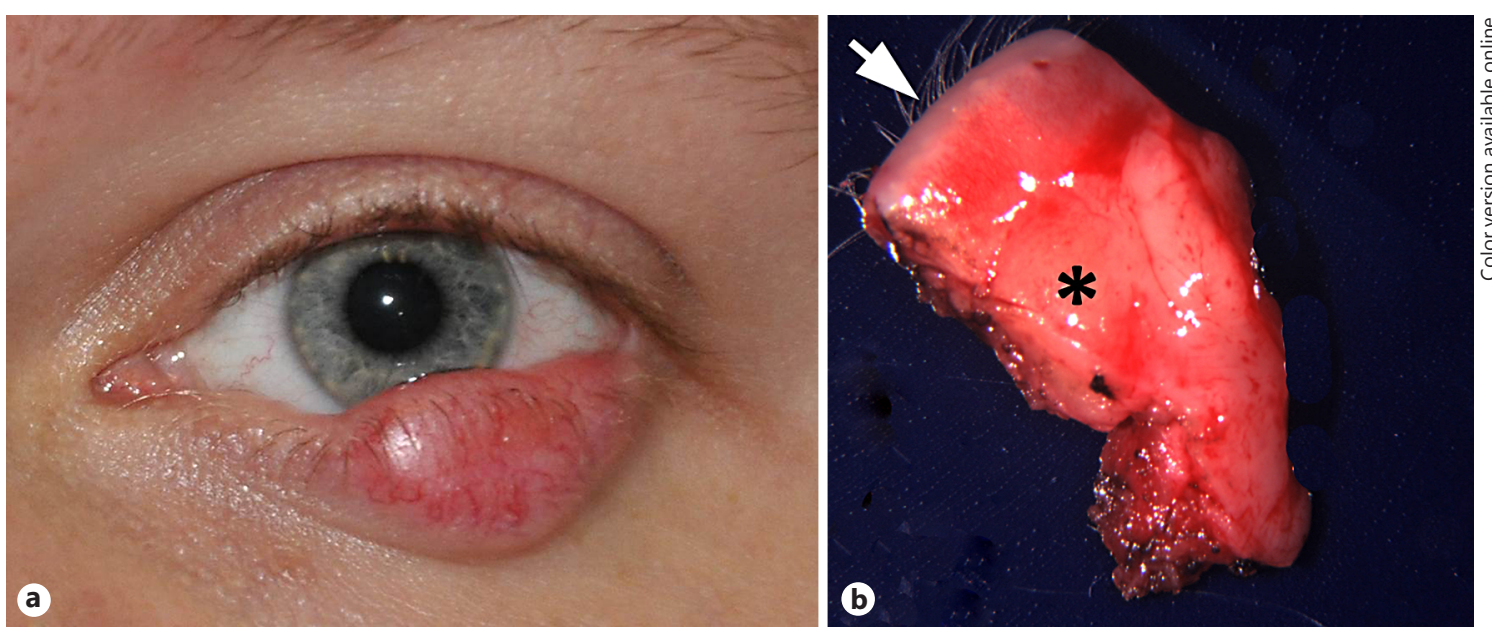

Fig. 1. a External photograph of the left eye demonstrates a vascular, erythematous mass in the central lower eyelid, associated with distortion of the eyelid margin architecture and focal madarosis. $\mathbf{b}$ Wedge resection of the eyelid (eyelid margin designated by an arrow) demonstrates a lobulated mass (asterisk) elevating the conjunctiva, at the junction of the tarsal plate and forniceal eyelid tissue. 
margin. The neoplasm appeared to originate from the accessory lacrimal gland of Wolfring, where the in situ component was seen (Fig. 2a). The neoplastic cells infiltrated the conjunctival substantia propria without epithelial involvement and focally replaced the apocrine glands of Moll without well-defined in situ component. The tumor cells were predominantly arranged in ductules with focal luminal eosinophilic secretory material and occasional mucin. The neoplastic cells had round nuclei with mild to moderate nuclear pleomorphism, focally prominent nucleoli, abundant eosinophilic cytoplasm with foci of apocrine-type secretion, and scattered intracytoplasmic vacuoles containing eosinophilic secretory material (Fig. 2b-d). No mucocytes or squamoid foci were noted. Although multifocal perineural invasion was seen, the neoplasm lacked the high-grade nuclear pleomorphism, brisk mitotic activity, and necrosis. Immunohistochemical studies showed that the neoplastic cells were diffusely positive for cytokeratin 7, BRST-2 (GCDFP-15), and androgen receptors (AR) (strong, diffuse nuclear staining), and focally positive for carcinoembryonic antigen. The neoplastic cells were negative for cytokeratin 20, p63, S-100, calponin, estrogen and progesterone receptors (ER, PR), prostate specific antigen (PSA), and thyroid transcription factor-1 (TTF-1) (Fig. 3). Although there was focal moderately intense $(2+)$ circumferential membranous immunoreactivity for HER $2 /$ neu, this pattern was seen in less than $30 \%$ of the cells, and the corresponding fluorescence in situ hybridization (FISH) studies showed no evidence of HER2/neu amplification. FISH studies for ETV6 and MAML2 rearrangements, using breakapart probes, were negative. The combined morphologic, immunohistochemical, and molecular genetic findings were interpreted as most compatible with adenocarcinoma, not otherwise specified, arising in an accessory lacrimal gland.

Following the surgery, the patient underwent systemic oncologic workup, including the MRI and whole-body PET/CT scan, all of which were negative for another primary tumor and metastatic disease. Four years following the surgery, the patient remains in good health, with no evidence of local recurrence, regional metastases, or distant metastatic disease.
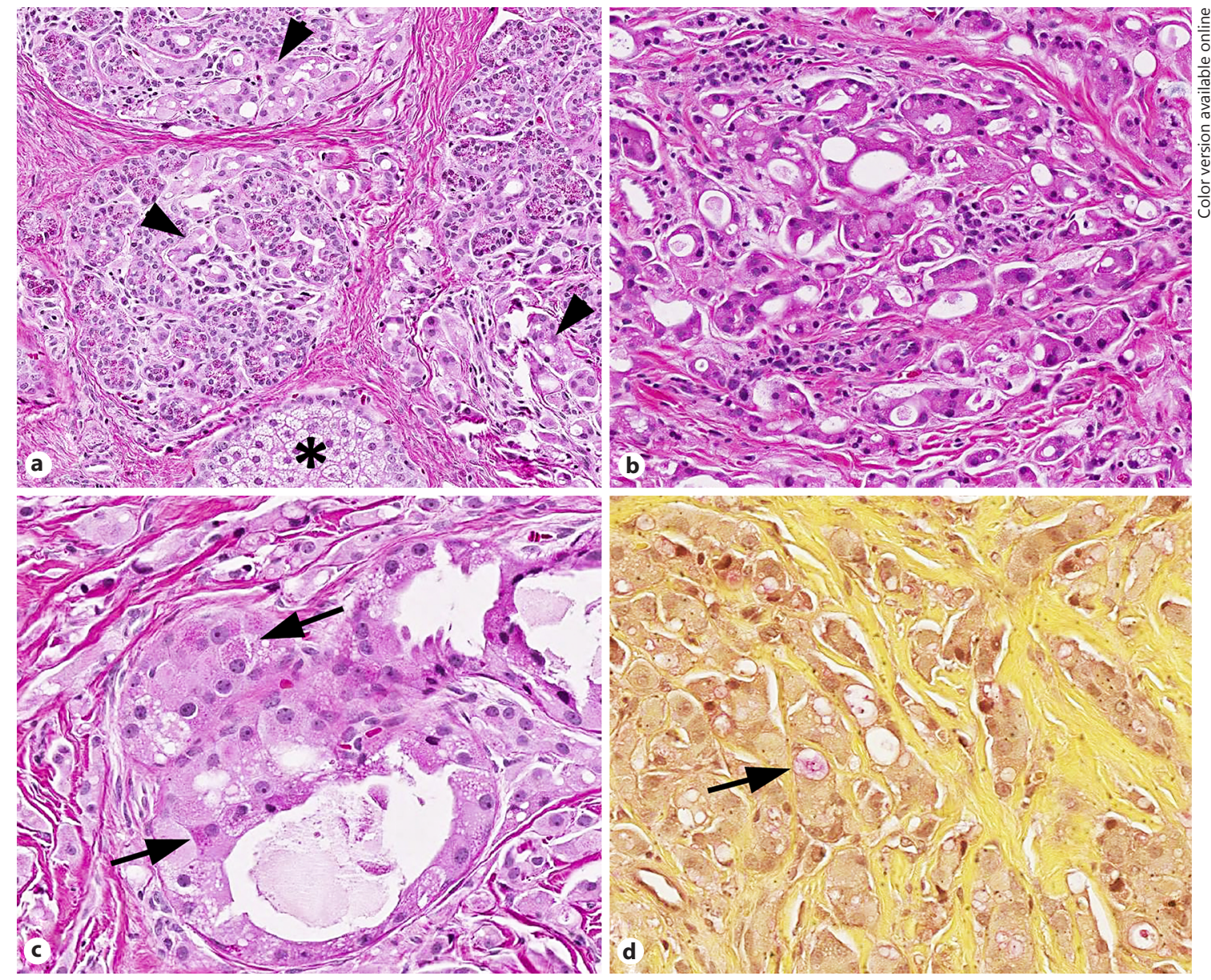

Fig. 2. Histopathologic features of the mass. a Low-power photomicrograph demonstrates accessory lacrimal gland lobules with an in situ proliferation of atypical eosinophilic cells (arrowheads) adjacent to the Meibomian glands (asterisk). b The neoplastic cells have abundant eosinophilic cytoplasm with focal intracytoplasmic vacuoles and are arranged in ductules with eosinophilic secretory material. c Secretory granules are focally noted (arrows). d Mucicarmine stain highlights rare foci of mucin. Stains: hematoxylineosin (a-c), mucicarmine (d); original magnification $\times 25(\mathbf{a}), \times 50$ (b), $\times 100(\mathbf{c}, \mathbf{d})$.
Adenocarcinoma, Not Otherwise

Specified, of Accessory Lacrimal Gland
Ocul Oncol Pathol 2018;4:67-72

DOI: $10.1159 / 000478730$ 


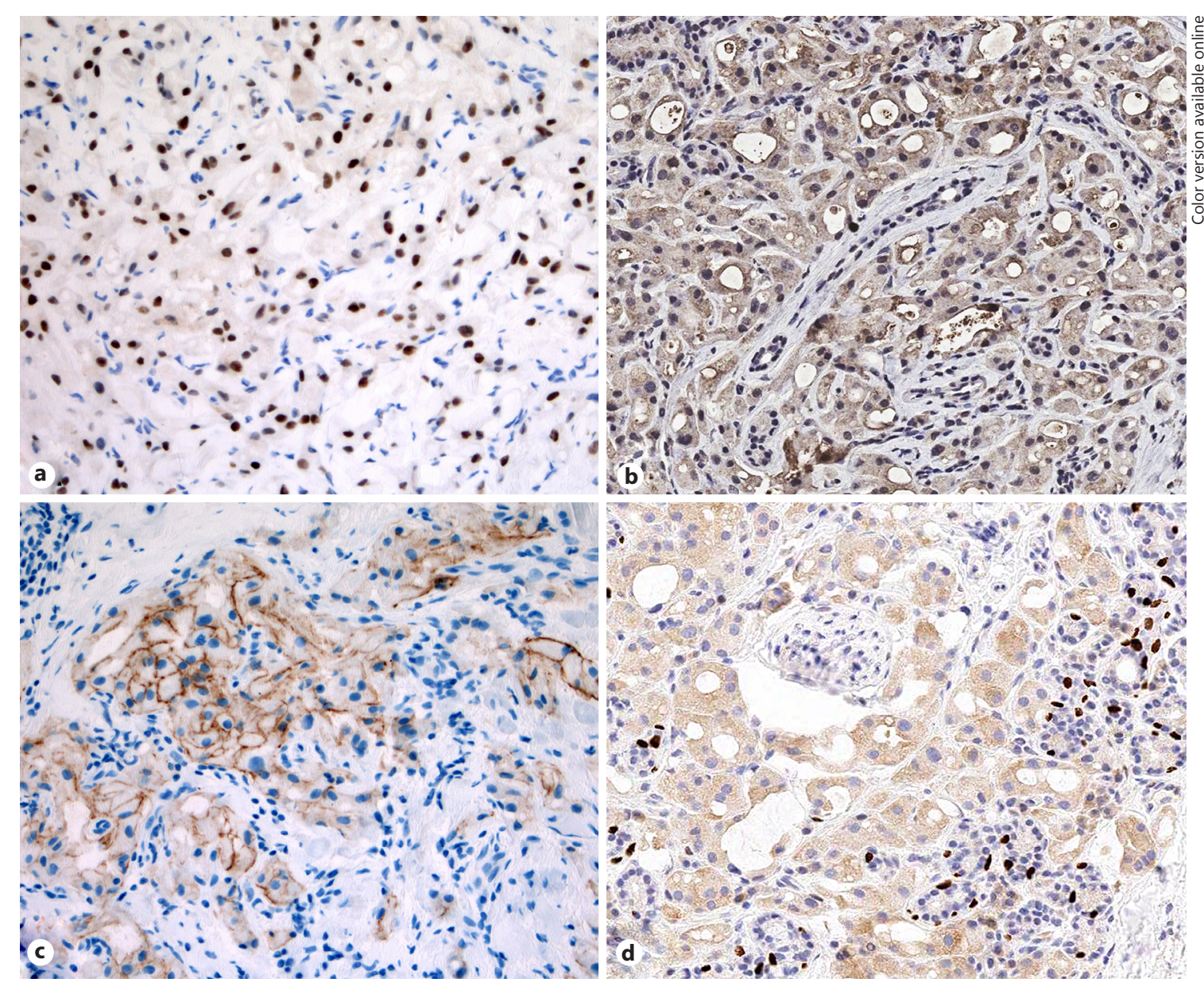

Fig. 3. Immunohistochemical findings. a The neoplastic cells demonstrate diffuse and strong nuclear immunoreactivity for androgen receptors (AR). b There is diffuse cytoplasmic immunoreactivity for BRST-2 (GCDFP-15). c Focal moderate (2+) membranous immunoreactivity for HER $2 /$ neu is seen. d No nuclear immunoreactivity for p63 is noted, with intact nuclear immunoreactivity in the myoepithelial cells of the uninvolved lacrimal gland. Antibodies: AR (a), BRST-2 (b), HER2 (c), p63 (d); all figures, original magnification $\times 25$.

\section{Discussion}

Primary malignant epithelial tumors of the lacrimal gland are rare, but those arising in the accessory lacrimal glands are rarer still, with less than 10 cases described in the literature [1-5]. The rarity of these lesions necessitates adoption of the continually evolving and diversifying classification scheme for the salivary gland tumors. This successful application of salivary gland classification to the lacrimal gland neoplasms is reflected in a decline in frequency of primary lacrimal gland adenocarcinoma from $10 \%$ of all malignant lacrimal gland tumors in a 2003 review by Shields et al. [6] to 5\% in a classification reappraisal by Weis and colleagues in 2009 [7]. Since that time, the advances in the understanding of molecular pathogenesis of the salivary gland neoplasia have led to emergence of new diagnostic entities, which are reflected in the most recent 4th edition of the World Health Organization Classification of Head and Neck Tumours [8].

We attempted to apply the current diagnostic guidelines to characterize our patient's neoplasm. The main differential diagnosis of this tumor included the primary accessory lacrimal gland neoplasms with glandular or microcystic differentiation and apocrine and oncocytic features, such as salivary duct carcinoma, mammary analogue secretory carcinoma (MASC), oncocytic variant of mucoepidermoid carcinoma, oncocytic carcinoma, and acinic cell carcinoma. Morphologically, the absence of an identifiable oncocytoma component and lack of appreciable acinar differentiation in conjunction with immu- 
noreactivity of the neoplastic cells for AR, BRST-2, and HER2/neu argues against oncocytic carcinoma and acinic cell carcinoma [9]. Though oncocytic mucoepidermoid carcinoma was a morphologic consideration, this neoplasm demonstrated prominent ductular differentiation and lacked immunoreactivity for p63, which is uniformly expressed in oncocytic mucoepidermoid carcinomas reported thus far $[9,10]$. Additionally, FISH studies for MAML2 rearrangement, observed in $40-84 \%$ of mucoepidermoid carcinomas, were negative [8]. Our tumor bore some morphologic similarity to MASC, a recently identified salivary gland neoplasm that frequently, though not consistently, expresses BRST-2, mammoglobin, and S-100. However, ETV6-NTRK3 translocation, which is diagnostic for MASC, was not observed in the neoplasm described here $[8,11]$. The diagnosis of salivary (lacrimal) duct carcinoma was also considered. The salivary duct carcinoma, or ductal carcinoma of the lacrimal gland, usually demonstrates apocrine differentiation in conjunction with AR immunoreactivity, and has been found to additionally express BRST-2, HER2/neu and, less frequently, ER and PR [9, 12]. However, salivary duct carcinoma is characteristically a high-grade neoplasm, with marked nuclear pleomorphism, brisk mitotic activity, and comedonecrosis, which were not observed in this case $[9,12]$. Additionally, salivary duct carcinoma and lacrimal gland ductal carcinoma tend to have a biologically aggressive clinical course which, thus far, has not been evident in our patient $[9,12,13]$.

Other diagnostic considerations also included metastatic lesions, primarily from the lung, prostate, and breast. Absence of immunoreactivity of the tumor for TTF-1 and PSA in conjunction with negative oncologic imaging studies argues against another primary malignancy. Finally, though we identified an in situ component of this tumor in the accessory lacrimal gland, which corresponded to the epicenter of the tumefaction, we cannot entirely exclude the possibility of the apocrine carcinoma originating in the glands of Moll with secondary involvement of the accessory lacrimal gland. Cutaneous apocrine carcinomas have a morphology and immunophenotype similar to our patient's tumor, although they have been described to express p63, ER, and PR, have patchy (as opposed to diffuse) positivity for CK7, and tend to lack HER2/neu expression [14-16].

Our patient describes an intriguing history of a chemical burn preceding evolution of the mass. However, while earlier epidemiologic studies have suggested that environmental and occupational exposures may be a risk factor in salivary gland cancer development, there is no conclusive evidence to support this association [17].

This report highlights the challenges involved in applying the salivary gland tumor classification to all accessory lacrimal gland neoplasms. Although at the first glance the diagnostic approach appears to be excessive, it is driven by the currently recognized differences in the biologic behavior of these lesions and in the emerging targeted therapies. For example, anti-androgen, anti-estrogen, and anti-HER2 therapies all have been utilized with varying success in patients with salivary duct carcinoma [18]. Thus, although we were unable to achieve a conclusive classification of this tumor, the workup provides potential avenues for targeted therapies in an eventuality of tumor recurrence or metastases.

\section{Statement of Ethics}

The Institution Ethics Review Committee approval was waved for this retrospective case report study. The study was performed in compliance with the tenets of the Declaration of Helsinki.

\section{Disclosure Statement}

None of the authors have relevant financial relationships with commercial interests.

\section{References}

Adenocarcinoma, Not Otherwise

Specified, of Accessory Lacrimal Gland
1 Duke TG, Fahy GT, Brown LJ: Adenoid cystic carcinoma of the superonasal conjunctival fornix. Orbit 2000;19:31-35.

2 Font RL, Del Valle M, Avedaño J, Longo M, Boniuk M: Primary adenoid cystic carcinoma of the conjunctiva arising from the accessory lacrimal glands: a clinicopathologic study of three cases. Cornea 2008;27:494-497.

3 Mendoza PR, Jakobiec FA, Krane JF: Immunohistochemical features of lacrimal gland epithelial tumors. Am J Ophthalmol 2013; 156:1147-1158
4 Johnson DS, Solomon AR, Washington CV: Mucoepidermoid/adenosquamous carcinoma of the skin: presentation of two cases. Dermatol Surg 2001;27:1046-1048.

5 Dithmar S, Wojno TH, Washington C, Grossniklaus HE: Mucoepidermoid carcinoma of an accessory lacrimal gland with orbital invasion. Ophthal Plast Reconstr Surg 2000;16: 162-166. 
6 Shields JA, Shields CL, Epstein JA, Scartozzi R, Eagle RC Jr: Review: primary epithelial malignancies of the lacrimal gland: the $2003 \mathrm{Ra}-$ mon L. Font lecture. Ophthal Plast Reconstr Surg 2004;20:10-21.

7 Weis E, Rootman J, Joly TJ, Berean KW, AlKatan HM, Pasternak S, Bonavolontà G, Strianese D, Saeed P, Feldman KA, Vangveeravong S, Lapointe JS, White VA: Epithelial lacrimal gland tumors: pathologic classification and current understanding. Arch Ophthalmol 2009;127:1016-1028.

8 Seethala RR, Stenman G: Update from the 4th Edition of the World Health Organization Classification of Head and Neck Tumours: Tumors of the Salivary Gland. Head Neck Pathol 2017;11:55-67.

9 Zhu S, Schuerch C, Hunt J: Review and updates of immunohistochemistry in selected salivary gland and head and neck tumors. Arch Pathol Lab Med 2015;139:55-66.
10 Weinreb I, Seethala RR, Perez-Ordoñez B, Chetty R, Hoschar AP, Hunt JL: Oncocytic mucoepidermoid carcinoma: clinicopathologic description in a series of 12 cases. Am J Surg Pathol 2009;33:409-416.

11 Stevens TM, Kovalovsky AO, Velosa C, Shi Q, Dai Q, Owen RP, Bell WC, Wei S, Althof PA, Sanmann JN, Sweeny L, Carroll WR, Siegal GP, Bullock MJ, Brandwein-Gensler M: Mammary analog secretory carcinoma, lowgrade salivary duct carcinoma, and mimickers: a comparative study. Mod Pathol 2015;28: 1084-1100.

12 Udager AM, Chiosea SI: Salivary Duct Carcinoma: An Update on Morphologic Mimics and Diagnostic Use of Androgen Receptor Immunohistochemistry. Head Neck Pathol 2017, DOI: 10.1007/s12105-017-0798-x (Epub ahead of print).

13 Kubota T, Moritani S, Ichihara S: Clinicopathologic and immunohistochemical features of primary ductal adenocarcinoma of lacrimal gland: five new cases and review of literature. Graefes Arch Clin Exp Ophthalmol 2013;251:2071-2076.
14 Schweitzer J, Vermeesch J, Zaleski T, Iacco M, Krach K, Ghaferi J, Cotton J: Primary cutaneous apocrine carcinoma: 2 cases and review of the pertinent histologic findings. JAAD Case Rep 2016;2:411-414.

15 Figueira EC, Danks J, Watanabe A, Khong JJ, Ong L, Selva D: Apocrine adenocarcinoma of the eyelid: case series and review. Ophthal Plast Reconstr Surg 2013;29:417-423.

16 Fernandez-Flores A: Immunohistochemical and morphologic evaluation of primary cutaneous apocrine carcinomas and cutaneous metastases from ductal breast carcinoma. Rom J Morphol Embryol 2012;53:879-892.

17 Zheng W, Shu XO, Ji BT, Gao YT: Diet and other risk factors for cancer of the salivary glands: a population-based case-control study. Int J Cancer 1996;67:194-198.

18 Keller G, Steinmann D, Quaas A, Grünwald V, Janssen S, Hussein K: New concepts of personalized therapy in salivary gland carcinomas. Oral Oncol 2017;68:103-113. 